\title{
Up-regulation of miR-24-1-5p is involved in the chemoprevention of colorectal cancer by black raspberry anthocyanins
}

\author{
He Zhang ${ }^{1} \dagger$, Jun Guo ${ }^{1} \dagger$, Liping $\mathrm{MaO}^{1}$, Qianqian $\mathrm{Li}^{1}$, Mengnan $\mathrm{Guo}^{1}$, Teng $\mathrm{Mu}^{1}$, Qiuhua Zhang ${ }^{2}$ and \\ Xiuli $\mathrm{Bi}^{1,3 *}$ \\ ${ }^{1}$ College of Life Science, Liaoning University, Shenyang 110036, People's Republic of China \\ ${ }^{2}$ Department of Pharmacology, Liaoning University of Traditional Chinese Medicine, Shenyang 110036, People's Republic \\ of China \\ ${ }^{3}$ Research Center for Computer Simulating and Information Processing of Bio-macromolecules of Liaoning Province, \\ Liaoning University, Shenyang 110036, People's Republic of China
}

(Submitted 14 May 2018 - Final revision received 28 September 2018 - Accepted 4 October 2018; First published online 17 December 2018)

\begin{abstract}
As important epigenetic regulators, microRNA regulate protein expression by triggering the degradation of target mRNA and/or by inhibiting their translation. Dysregulation of microRNA expression has been reported in several cancers, including colorectal cancer. In this study, microRNA-array differential analysis revealed strongly enhanced expression of miR-24-1-5p in the colon tissue of azoxymethane/dextran sulphate sodium-induced mice that were fed with black raspberry anthocyanins for 9 weeks. Overexpression of miR-24-1-5p in human colorectal cancer cells significantly repressed $\beta$-catenin expression, and simultaneously decreased cell proliferation, migration and survival. Furthermore, miR-24-1-5p could target $\beta$-catenin and trigger a negative regulatory loop for $\beta$-catenin and its downstream target genes. $\beta$-Catenin signalling is vital to the formation and progression of human colorectal cancer. The current findings therefore identified miR-24-1-5p as a potent regulator of $\beta$-catenin, and this may provide a novel chemopreventive and therapeutic strategy for $\beta$-catenin signalling-driven colorectal cancer.
\end{abstract}

Key words: miR-24-1-5p: Black raspberry anthocyanins: Azoxymethane/dextran sulphate sodium: Colorectal cancer: $\beta$-Catenin

Colorectal cancer (CRC) is the third most malignant tumour worldwide, accounting for approximately $10 \%$ of all cancer incidence and mortality ${ }^{(1,2)}$. Despite the considerable effort dedicated to CRC diagnosis and therapeutic strategy over the past two decades, one-fifth of the patients are still being diagnosed with CRC in the advanced stage, which is usually treated by a combination of surgery and adjuvant chemotherapy ${ }^{(3-5)}$. Most patients are resistant to chemotherapy, and the cancer may occur again in these patients with eventual metastasis ${ }^{(6)}$. A number of recent studies have reported significant association of microRNA (miRNA) expression with the progression of $\mathrm{CRC}^{(7-9)}$. Therefore, based on RNA network analysis, it would be desirable to search for a novel targets that can be used in the prevention or therapy of CRC.

miRNA are endogenous small non-coding RNA with 18-22 nt in length, and they regulate gene expression by interfering with the translation of mRNA through binding to their 3'-UTR (untranslated regions), directly degrading the bound
$\mathrm{mRNA}^{(10,11)}$. Aberrant expression of miRNA can give rise to either tumour suppressors or oncogenes in many human cancers. In CRC, several miRNA have been identified as essential for tumour development. The differential expression of miRNA in human CRC tissues and normal colorectal mucosa was first reported by Michael et $a l^{(12)}$, and twenty-eight differentially expressed miRNA were detected, including miR-320, miR-321, miR-200c, miR-223 and miR-145. Monzo et al. ${ }^{(13)}$ found that miR-17-5p can target E2F transcription factor 1 present in the early embryonic development and tumourigenesis of human colon, regulating cell proliferation in both cases. Other investigators have reported that miR-34a directly and negatively controls a series of downstream target genes of $p 53$, forming a miR-34a-p53 positive feedback loop ${ }^{(14)}$. Bandres et al. ${ }^{(15)}$ found that miRNA-451 can regulate the expression of migration inhibitory factor $(M I F)$, thereby, inhibiting the proliferation of $\mathrm{CRC}$ cells and enhancing the sensitivity of the tumour to radiotherapy. In addition, miRNA-320a and miR-139 can target

Abbreviations: AOM, azoxymethane; BRB, black raspberry; CDK4, cyclin-dependent kinase 4; CRC, colorectal cancer; DSS, dextran sulphate sodium; miRNA, microRNA; qRT-PCR, quantitative RT-PCR.

*Corresponding author: X. Bi, email xiulibi@gmail.com, xiulibi@lnu.edu.cn

$\dagger$ These authors contributed equally to this work. 
$\beta$-catenin and RAP1B (member of the RAS oncogene family), respectively, to inhibit the proliferation of human colon cancer cells $^{(16,17)}$. miR-24 was first discovered by Lagos-Quintana et $a l^{(18)}$ in 2001 when non-spine animals and spine animals were studied. The human miR-24 consists of miR-24-1 and miR-24-2, and are located on chromosomes 9 and 19, respectively, and the mature sequences include miR-24-3p, miR-24-1$5 p$ and miR-24-2-5 $\mathrm{p}^{(19)}$. Aberrant expression of miR-24-1 in several types of cancer tissues has been reported ${ }^{(20-26)}$. However, the functional role of miR-24-1-5p in CRC is still unknown.

Black raspberry (BRB) belongs to the Rubus occidentalis families of berries, and they are rich in flavonoids and phenolic acids $^{(27,28)}$. The chemopreventive effect of BRB against CRC has been well documented by studies carried out by our research group and those of others ${ }^{(29-32)}$. However, few studies have focussed on the role of miRNA in association with the chemopreventive activity of $\mathrm{BRB}$ in $\mathrm{CRC}$ and the underlying mechanisms.

In this study, we hypothesised that miR-24-1-5p might play an important role in the initiation and progression of $\mathrm{CRC}$, and that it might also be involved in the chemopreventive effect of BRB against CRC. To test this hypothesis, we conducted one in vivo study with mice and several in vitro studies with human cell lines, and found that miR-24-1-5p could function as a tumour suppressor by targeting several $\beta$-catenin-related oncogenes involved in specific cancer-related pathways in CRC. Our current finding might facilitate the development of novel diagnostic and therapeutic strategies for the prevention and treatment of CRC.

\section{Methods}

\section{Chemicals and reagents}

Azoxymethane (AOM, A5486) was purchased from SigmaAldrich Inc. Dextran sulphate sodium (DSS, 0216011091) was purchased from MP Inc. Jianfeng Nature Products Technology Co., Ltd provided the BRB anthocyanins (purity >90\%). BRB anthocyanins consist of three major anthocyanins cyanidinglucoside, cyanidin-xylosylutinoside and cyanidin-rutinoside, and the contents are $2.63,0.73$ and $16.91 \mathrm{mg} / \mathrm{g}$, respectively. The cyanidin-sambubioside is less abundant, the detail of which can be found in our recently published paper ${ }^{(33)}$. $\beta$-Catenin, cyclin D1, c-Myc and cyclin-dependent kinase 4 (CDK4) were obtained from Cell Signaling Technology Inc. $\beta$-Actin, E-cadherin, glycogen synthase kinase $3 \beta$ (GSK3- $\beta$ ), phosphoglycogen synthase kinase $3 \beta$ (p-GSK3- $\beta$ ), B-cell lymphoma2 (BCL-2) and haemagglutinin were obtained from Sangon Biotech Co., Ltd. Secreted frizzled-related protein 2 (SFRP2) was bought from Abcam Inc. Secreted frizzled-related protein 5 (SFRP5) was obtained from Biorbyt Explore Bioreagents. Goat anti-rabbit and goat anti-mouse IgG conjugated with horseradish peroxidase used as secondary antibodies in Western blot were obtained from ComWin Biotech Co., Ltd.

\section{Animal care and experimental protocol}

A total of 5-weeks-old male C57BL/6J mice (18-20g, Benxi Changsheng Laboratory Animal Technology Co., Ltd) were given water ad libitum, and maintained under a $12 \mathrm{~h}$ light-12 $\mathrm{h}$ dark cycle. The animals were housed in cages (five animals per cage) in a room with temperature set at $21 \pm 2 \cdot 0^{\circ} \mathrm{C}$ and humidity controlled at $50 \pm 5 \%$. All animal experiment protocols were approved by the Ethics Committee of Liaoning University of Traditional Chinese Medicine (Shenyang, China).

\section{Establishment of colitis-induced colorectal cancer mice model and black raspberry anthocyanins supplementation}

After 1 week of adaptation, the mice were given AOM (10 $\mathrm{mg} / \mathrm{kg}$ body weight) in a single intraperitoneal injection. In all, 1 week later, they were given $2 \%$ DSS in the drinking water for 1 week, followed by normal water for another 2 weeks, and this cycle was repeated two more times.

The mice were divided into three groups: one healthy control group and two AOM/DSS-treated groups. Each group consisted of ten animals. Mice in the healthy control group were not given any treatment, and they were fed with a chow diet over a 9-week period. As for the two AOM/DSS-treated groups, one group was fed with just the chow diet, whereas the other group was fed with the chow diet containing $7.0 \mu \mathrm{mol} / \mathrm{g}$ BRB anthocyanins (10\% BRB). The concentration of BRB anthocyanins in the diet corresponded to the anthocyanins content in a $10 \%$ freeze-dried $\mathrm{BRB}$ powder, and it was chosen on the basis of previous study $^{(32,34)}$. The formula composition of the chow diet and BRB anthocyanins-supplemented diet is listed in Table 1. The diets were stored at $-20^{\circ} \mathrm{C}$ before they were used in the experiment.

At the end of 9th week, the mice were sacrificed by $\mathrm{CO}_{2}$ inhalation followed by cervical dislocation. The colon tissue was immediately frozen in liquid $\mathrm{N}_{2}$, and then stored at $-80^{\circ} \mathrm{C}$ for miRNA array analysis.

\section{MicroRNA microarray expression profiling and data analysis}

miRNA expression profiles were generated using the Agilent Mouse miRNA $(8 \times 60 \mathrm{~K}$, design ID: 070155; Agilent Technologies Inc.). Total RNA was quantified by the NanoDrop

Table 1. Composition of chow diet and black raspberry (BRB) anthocyanins-supplemented diet

\begin{tabular}{lcc}
\hline Macronutrient composition & Chow diet & $\begin{array}{c}\text { BRB anthocyanins- } \\
\text { supplemented diet }\end{array}$ \\
\hline Protein (\% of energy) & 20 & 20 \\
Fat (\% of energy) & 10 & 10 \\
Carbohydrate (\% of energy) & 70 & 70 \\
Energy (MJ $/ \mathrm{kg})$ & $18 \cdot 8$ & $18 \cdot 8$ \\
Ingredients & & \\
Soyabean (g/kg) & 56 & 56 \\
Maize starch (g/kg) & 300 & 300 \\
Flour (g/kg) & 250 & 250 \\
Bran (g/kg) & 80 & 80 \\
Fish meal (g/kg) & 258 & 258 \\
Fibre (cellulose) (g/kg) & 40 & 40 \\
Ca (g/kg) & 10 & 10 \\
P (g/kg) & 6 & 6 \\
BRB anthocyanins (mol $/ \mathrm{kg})$ & - & 7 \\
\hline
\end{tabular}


ND-2000 (Thermo Scientific). RNA integrity was assessed by an Agilent Bioanalyzer 2100 (Agilent Technologies Inc.). Total RNA was dephosphorylated, denaturated and then labelled with cyanine-3-cytidine triphosphate. After purification, the labelled RNA was hybridised to the microarray. After washing, the array was scanned with an Agilent Scanner G2505C (Agilent Technologies Inc.). Image of the array was acquired by Feature Extraction software (version 10.7.1.1; Agilent Technologies Inc.). Genespring software (version 13.1; Agilent Technologies Inc.) was used to analyse the raw data.

\section{RNA extraction and quantitative real-time $P C R$}

Total RNA was isolated from the cells using a TRIzol Reagent Kit (ComWin Biotech Co., Ltd), and the first-strand complementary DNA (cDNA) encoding the miRNA with poly A was generated by reverse transcription using a miRNA cDNA synthesis kit (ComWin Biotech Co., Ltd). Real-time PCR (RT-PCR) was performed using SYBR Green assay with specific primers. The assay was conducted with a miRNA real-time assay kit (ComWin Biotech Co., Ltd) and amplification was performed on an Applied Biosystems 7500 real-time PCR system in accordance with the manufacturer's instructions. The sequences of the primers were: mmu-miR-24-1-5p: forward 5'-AGTGCCTACT GAGCTGATATCAGT-3'; hsa-miR-24-1-5p: forward 5'-GTGCCT ACTGAGCTGATATCAGT- $3^{\prime} ; \beta$-catenin: forward $5^{\prime}$-GCTGGTG ACAGGGAAGACAT- $3^{\prime}$, reverse 5'-AGGCAAACCGTGAAAA GATG-3'; cyclin D1: forward 5'-AACTACCTGGACCGCTTCCT-3', reverse 5'-CCACTTGAGCTTGTTCACCA-3'; c-Myc: forward 5'-TTCGGGTAGTGGAAAACCAG-3'， reverse 5'-CAGCAGCTCG AATTTCTTCC-3'; CDK4: forward 5'-ATTGGTGTCGGTGCCT ATG-3', reverse 5'-AACTGTGCTGATGGGAAGG-3'. The expression levels of these genes were normalised to that of the internal control (U6: forward 5'-ATTGGAACGATACAGAG AAGATT-3', reverse 5 -GGAACGCTTCACGAATTTG- $3^{\prime}$ or $\beta$-actin: forward 5'-AGGCAAACCGTGAAAAGATG-3'， reverse 5'-AGGCAAACCG TGAAAAGATG- $3^{\prime}$ ) according to the $2^{-\Delta \Delta C_{\mathrm{T}}}$ method.

\section{Plasmid constructs and transfection}

The mature sequence of miR-24-1-5p is UGCCUACUGAGCUGAUAUCAGU. The sequence of human pri miR-24-1-5p was constructed using the following primers: miR-24-1-5p forward, 5'-CCGCTCGAGCAACAGGGTTTTCCAAGTCTAC-3' and miR-24-1-5p reverse, 5'-GGAAGATCTTCACCTAAGTCGGAAAT CATGTGGTA- $3^{\prime}$. The PCR product was digested with $X h o$ I and $B g / I I$ and then inserted into XhoI-BglII cut pcDNA3haemagglutinin. HCT-116 and Caco-2 cells were then transfected with the human pri-miR-24-1-5p using lipofectamine 2000 (Invitrogen). Cells transfected with the blank plasmid were used as negative controls. The constructed human pri-miR-24-1$5 \mathrm{p}$ was validated by sequencing.

\section{Cell culture}

Human CRC cell lines HCT-116 (CCL-247; American Type Culture Collection) and Caco-2 (Cell Bank of Beijing Cancer Institute) were cultured in Dulbecco's modified Eagle's medium and $\alpha$-minimum essential medium (Hyclone, Thermo Fisher Scientific Inc.), respectively. In both cases, the medium was supplemented with $10 \%(\mathrm{v} / \mathrm{v})$ fetal bovine serum (Dalian Meilun Biology), $10000 \mathrm{U} / \mathrm{ml}$ penicillin $\mathrm{G}, 10 \mathrm{mg} / \mathrm{ml}$ streptomycin, and the cultures were incubated in a $5 \% \mathrm{CO}_{2}$ humidified atmosphere at $37^{\circ} \mathrm{C}$.

\section{Wound healing assay}

HCT-116 and Caco-2 cells with 70\% confluency were used in the wound healing assay. First, a sterile pipette tip was used to make a straight-line scratch in the cell monolayer. The cells were washed with PBS followed by the addition of fresh medium. They were then transfected with miR-24-1-5p, and after $24 \mathrm{~h}$, the medium was discarded, and the gap remaining in the scratch was calculated by photomicrograph.

\section{Cell proliferation and colony formation assays}

Cell viability was assessed by the 3-(4, 5-dimethylthiazol-2-yl)2,5-diphenyltetrazolium bromide (MTT) assay. Briefly, $24 \mathrm{~h}$ after transfection with miR-24-1-5p, the Caco-2 and HCT-116 cells were incubated for $4 \mathrm{~h}$ with $0.5 \mathrm{mg} / \mathrm{ml}$ MTT reagent. The culture medium was then removed, and $100 \mu$ dimethyl sulfoxide was added to each well to dissolve the formazan crystals. The absorbance of the plate was measured at $490 \mathrm{~nm}$. To further investigate the role of miR-24-1-5p in cancer cell survival, the cells were seeded in six-well plates at 200/well and grown for 2 weeks for colony formation assay. The colonies of cells that appeared were first washed with PBS, and then fixed with methanol followed by staining with $0.5 \%$ Giemsa. The number of colonies appearing in each well was counted using an inverted microscope.

\section{Transwell migration assay}

Caco- 2 and HCT-116 cells were seeded on the top chamber of a transwell, and serum-free medium was added to the bottom chamber. After $24 \mathrm{~h}$, the cells on top of the membrane were removed with a cotton swab, and the membranes of the transwell inserts were then fixed with $4 \%$ paraformaldehyde. The cells that migrated to the bottom of the membrane were stained with 4',6-diamidino-2-phenylindole (Beijing Dingguo Changsheng Biotechnology Co., Ltd). A total of ten fields were randomly selected from each insert and captured under a microscope at $20 \times$ magnification. Cell numbers were quantified using Image-Pro Plus 6.0. To calculate the migration index, the number of cells obtained for each treated group was normalised to the number of cells in the control group.

\section{Target prediction of microRNA}

Target genes of miR-24-1-5p were predicted using algorithms, MiRanda version $3.3 \mathrm{a}$, with the default parameters and cutoffs (score $S \geq 140$ and energy $E \leq-7 \cdot 0$ ) and website tools (http:// www.microrna.org/microrna/home.do). 
Up-regulation of miR-24-1-5p

\section{Western blotting}

Cells were lysed in chilled radioimmunoprecipitation assay buffer containing $1 \mathrm{mmol} / \mathrm{l}$ phenylmethylsulfonyl fluoride (Beijing Dingguo Changsheng Biotechnology Co., Ltd) for $30 \mathrm{~min}$ on ice. After centrifugation at $14000 \mathrm{~g} / \mathrm{min}$ for $15 \mathrm{~min}$ at $4^{\circ} \mathrm{C}$, the supernatant was collected and a sample of the supernatant containing $40 \mu \mathrm{g}$ protein was resolved by SDS-PAGE using $10 \%$ gel. After electrophoresis, the proteins in the gel were transferred to a nitrocellulose membrane (Sangon Biotech Co., Ltd), and the membrane was blocked with $5 \%$ bovine serum albumin fraction $\mathrm{V}$ (Roche) in Tris-buffered saline and Tween 20 buffer for $2 \mathrm{~h}$. It was then incubated with the appropriate primary antibody for $3 \mathrm{~h}$ at room temperature followed by the corresponding secondary antibody. Signals on the blot were detected by an enhanced chemiluminescence technique (Amersham Life Science).

\section{Immunohistochemical staining}

Mouse intestinal tissues from AOM/DSS-induced mice with or without BRB anthocyanins were collected, fixed in $10 \%$ buffered formalin and embedded in paraffin as previously reported $^{(35)}$. Sections ( $4 \mu \mathrm{m}$ thick) of the tissue were used for immunohistochemical staining using anti-E-cadherin antibody (Santa Cruz Biotechnologies). The procedure was similar to that previously reported $^{(35)}$. In brief, the sections were deparaffined, rehydrated, quenched with $3 \% \mathrm{H}_{2} \mathrm{O}_{2}$, blocked with $10 \%$ normal serum and then incubated with $\beta$-catenin antibody for overnight at $4^{\circ} \mathrm{C}$ followed by incubation with secondary antibody and avidin-biotin complex (ABC kit; Vector Laboratory). The staining was developed using 3',5'-diaminobenzidine followed by lightly counter-staining with haematoxylin. Image of the stained slide was captured by the Aperio Image system.

\section{Statistical methods}

The statistical software SPSS 19.0 was used to analyse the data. To compare differences within and between groups, $t$ tests and ANOVA followed by Fisher's least significant difference analysis were used. Statistical significance was considered at the $P<0.05$ level.

\section{Results}

\section{Tissue microRNA microarray profiling}

To elucidate the miRNA-mediated tumourgenic pathways in CRC, identifying the aberrantly expressed miRNA would be the first step. Based on this point, we performed tissue miRNA microarray analysis to obtain the global miRNA expression signatures in the colon tissue of AOM/DSS-induced mice with or without BRB anthocyanins supplementation. Comparison of the results from the two groups showed that several miRNA were differentially regulated by the administration of BRB anthocyanins (Fig. 1(a)). The result obtained from the hierarchical clustering analyses of the tissue arrays is presented in Fig. 1(b). Low level of miR-24-1-5p was expressed in the AOM/ DSS-induced group, but after 9 weeks of feeding with BRB anthocyanins, the level of mir-24-1-5p was significantly (a)

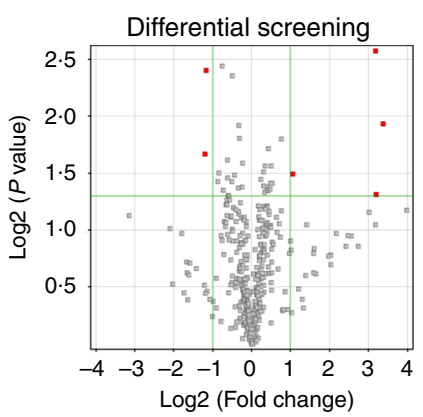

(b)
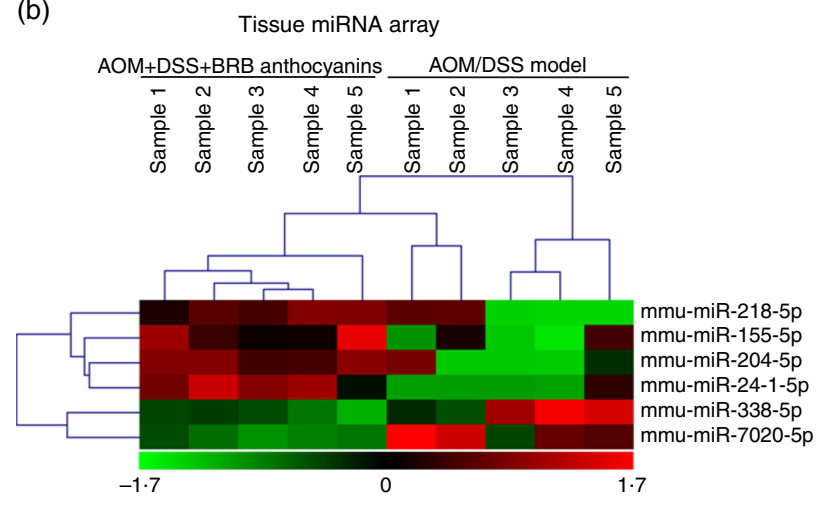

Fig. 1. Differential expression of microRNA (miRNA) in mice colon tissue. (a) Up-regulated miRNA are indicated in red. (b) Tissue miRNA array. Green down-regulation; red - up-regulation ( $n 5$ ). AOM, azoxymethane; DSS, dextran sulphate sodium; BRB, black raspberry.

up-regulated, suggesting that miR-24-1-5p might have a suppressive effect on tumour formation. This also confirmed the chemopreventive property of BRB anthocyanins.

Expression of miR-24-1-5p is down-regulated in the colon tissue of azoxymethane/dextran sulphate sodium-induced mice and up-regulated in human colorectal cancer cells lines treated with black raspberry anthocyanins

To further evaluate the result shown by the microarray analysis using mouse model, miR-24-1-5p expression in the colon tissue of the AOM/DSS-induced group with or without BRB anthocyanins administration was analysed by quantitative RT-PCR (qRT-PCR). The expression pattern of miR-24-1-5p obtained by qRT-PCR was consistent with the microarray data (Fig. 2(a)). The expression of miR-24-1-5p was also determined in several human CRC cell lines (Caco-2, HCT-116, Lovo, HT29 and SW480) after treatment with different doses of BRB anthocyanins. BRB anthocyanins caused the level of miR-24-1-5p to increase. The pattern of miR-24-1-5p expression in BRB anthocyanins-treated human cancer cells was consistent with the miRNA microarray and qRT-PCR data obtained for the colon tissues of mice fed with the BRB anthocyanins-supplemented diet (Fig. 2(b)).

\section{$\beta$-Catenin in azoxymethane/dextran sulphate sodium-induced mice is reduced by black raspberry anthocyanins}

To determine the effect of $\beta$-catenin in AOM/DSS-induced mice regulated by $\mathrm{BRB}$ anthocyanins in situ, we performed 
(a)
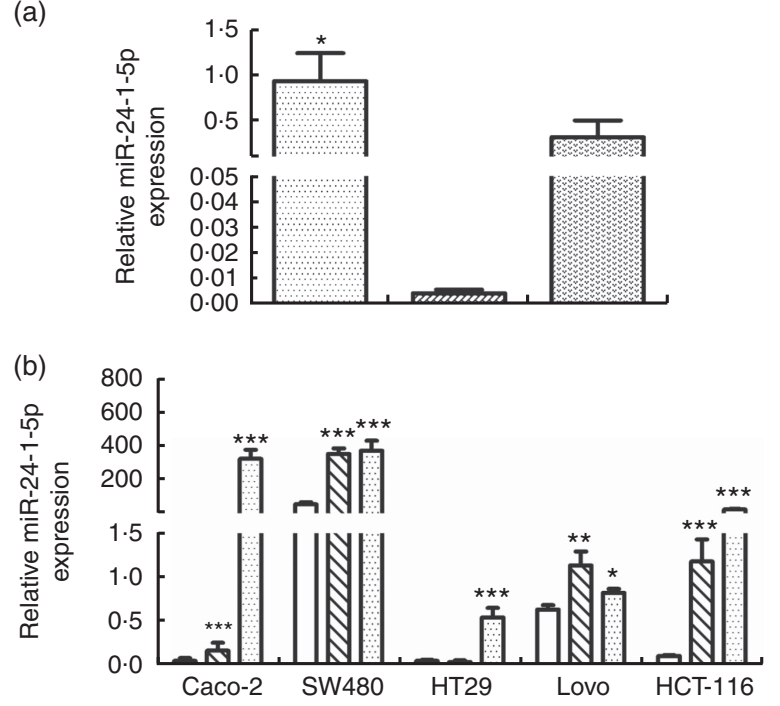

Fig. 2. miR-24-1-5p expression in colon tissue and colorectal cancer (CRC) cells as detected by quantitative RT-PCR (qRT-PCR). (a) qRT-PCR analysis of miR-24-1-5p levels in colon tissue of azoxymethane (AOM)/dextran sulphate sodium (DSS)-induced mice with or without black raspberry (BRB) anthocyanins supplement. (b) Relative miR-24-1-5p expression in different CRC cell lines. One-way ANOVA was used to compare different groups of human CRC cell lines or mouse colon tissues. Values are means $(n 5)$, with their standard errors represented by vertical bars. Mean values were significantly different when compared with the solvent control:

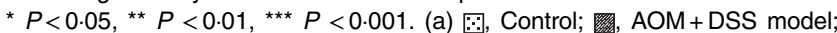
图, AOM + DSS + BRB anthocyanins. (b) $\square$, Control; $\Delta, 25 \mu \mathrm{g} / \mathrm{ml}$ BRB anthocyanins; $B, 50 \mu \mathrm{g} / \mathrm{ml} \mathrm{BRB}$ anthocyanins. immunohistochemical staining. AOM/DSS-induced mice that were given the BRB anthocyanins-supplemented diet showed significantly reduced levels of $\beta$-catenin in the small intestine compared with AOM/DSS-induced mice given the BRB anthocyanins-free diet (Fig. 3).

\section{Effects of restoring miR-24-1-5p expression on colorectal cancer cell proliferation and migration}

To further evaluate the biological function of miR-24-1-5p, human HCT-116 and Caco-2 cells were transfected with miR-24-1-5p and the viability of the cells was determined by MTT assay. Both miR-24-1-5p-transfected HCT-116 and Caco2 cells exhibited markedly reduced cell viability (56\% in the case of HCT-116 and $52 \%$ for Caco- 2 cells) compared with the corresponding control cells (Fig. 4(a) and (b)). These cells also displayed significantly reduced sclerosis as revealed by wound healing assay (Fig. 4(c) and (d)). Furthermore, they exhibited significant reduction in cell migration and cell survival as revealed by transwell assay (Fig. 4(e) and (f)) and colony formation assay (Fig. $4(\mathrm{~g})$ and (h)), respectively. Taken together, the results suggested that overexpression of miR-24-1-5p could significantly decrease the proliferation, migration and survival of these human CRC cell lines, thereby indicating that miR-24-1-5p might play a tumour-suppressive role in CRC by reducing or suppressing the growth of the tumour cells.
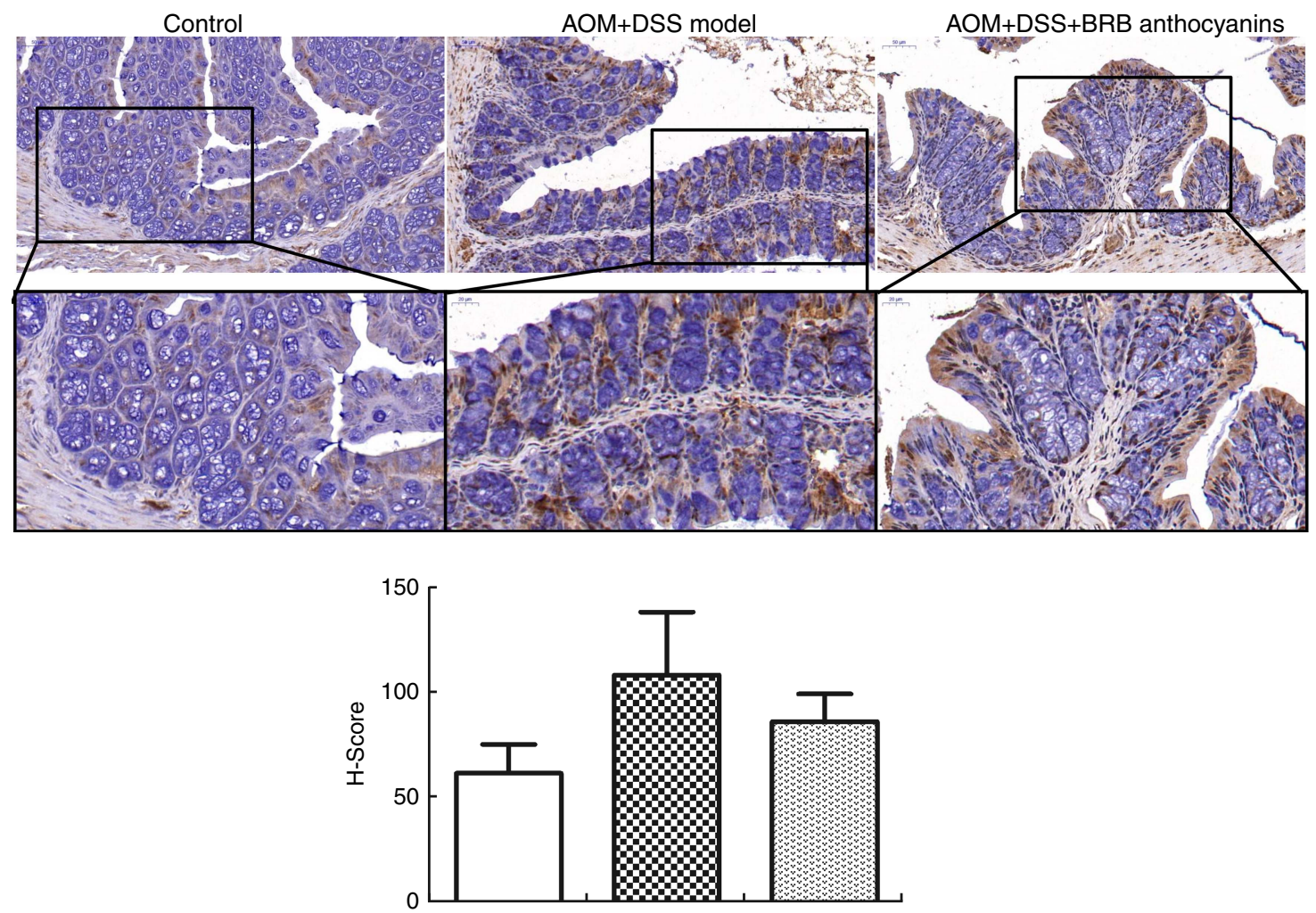

Fig. 3. Effect of black raspberry (BRB) anthocyanins supplement on $\beta$-catenin expression in mouse colon tissue. In situ $\beta$-catenin expression in the intestinal epithelial cells of azoxymethane (AOM)/dextran sulphate sodium (DSS)-induced mice with or without BRB anthocyanins supplement as detected by immnunohistochemical staining (anti- $\beta$-catenin antibody dilution 1:100) (magnification 20x and 40x, respectively). $\square$, Control; B, AOM + DSS model; 圈, AOM + DSS + BRB anthocyanins. 
Up-regulation of miR-24-1-5p
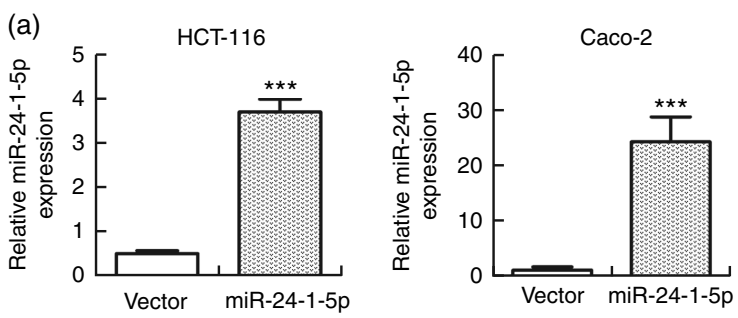

(c)
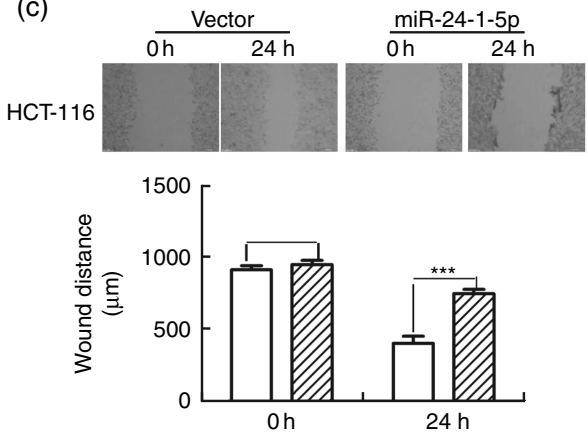

(e)

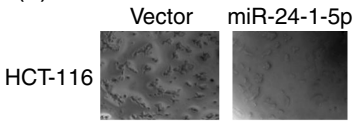

(f)
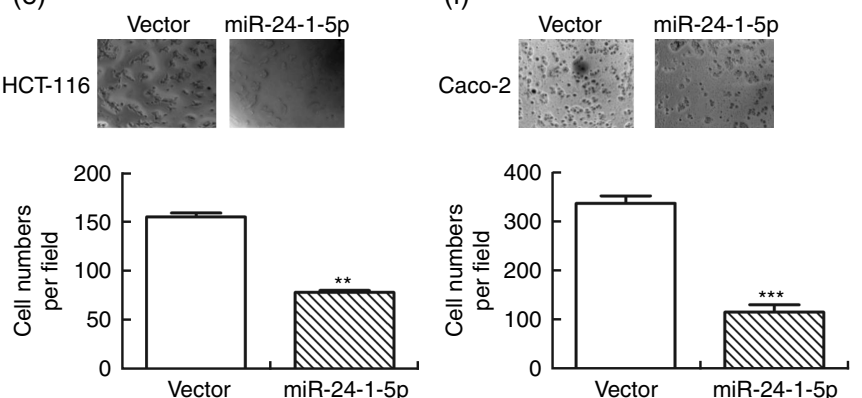
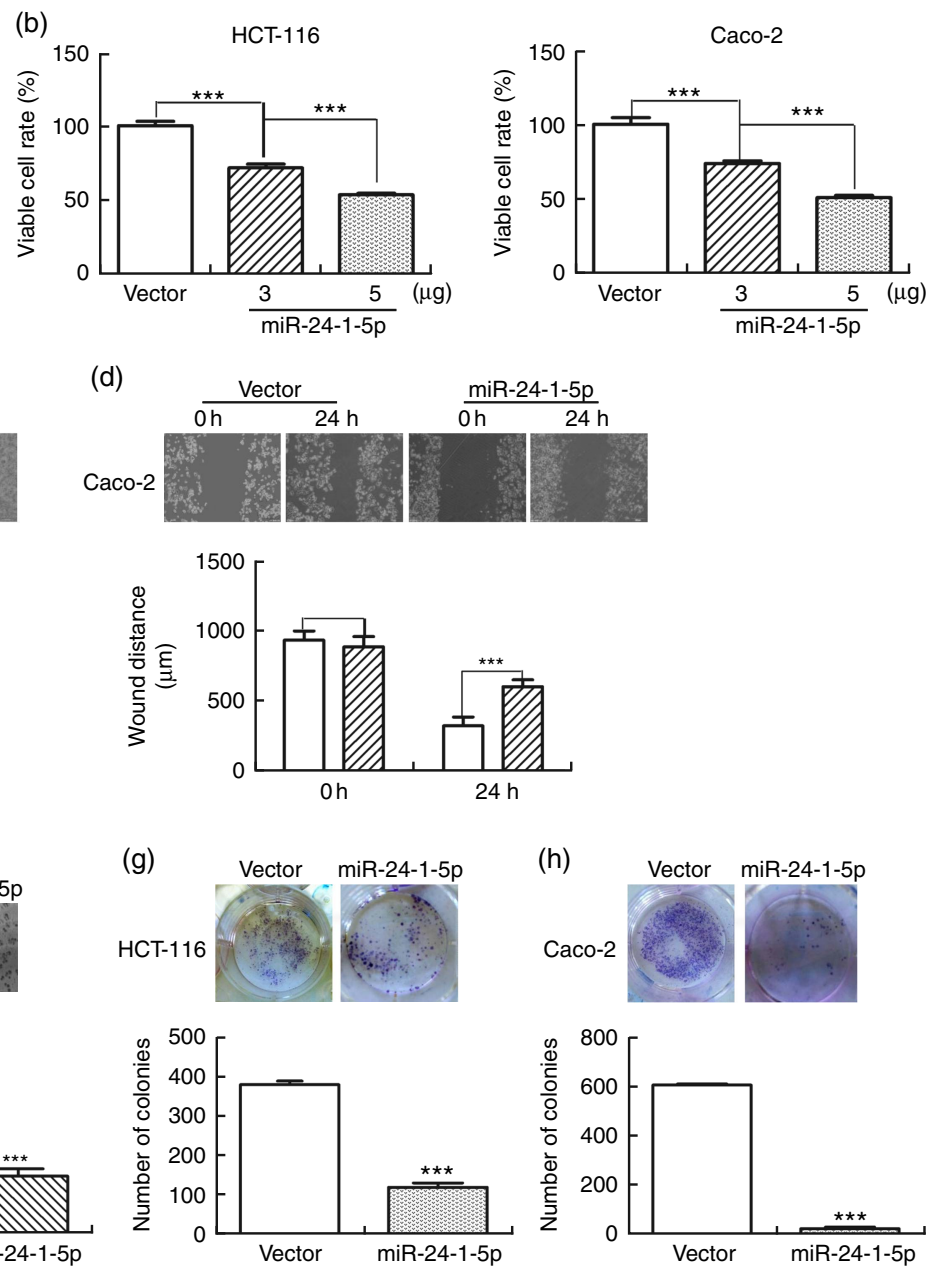

Fig. 4. miR-24-1-5p inhibits human colorectal cancer cells proliferation, migration and formation. HCT-116 and Caco-2 cells were transfected with $5 \mu$ g miR-24-1-5p for $24 \mathrm{~h}$ and then subjected to the following analyses: (a) expression level of miR-24-1-5p was measured by quantitative RT-PCR. (b) Cell viability was determined by 3-(4,5-dimethylthiazol-2-yl)-2,5-diphenyltetrazolium bromide assay. (c, d) Cell migration was evaluated by wound healing assay. The inhibitory effect on cell migration was determined by an increase in the percentage of the wound area remaining at $24 \mathrm{~h}$. Wound area at $0 \mathrm{~h}$ was taken as $100 \%$. $\square$, Vector; $Z$, miR-24-1-5p. (e, f) Cell invasion was examined by microscopy. $(\mathrm{g}, \mathrm{h})$ Representative micrographs and quantitative analysis of colony formation. Differences within and between groups were compared by one-way ANOVA followed by Fisher's least significant difference analysis. Values are means $(n 3)$, with their standard errors represented by vertical bars. Mean values were significantly different when compared with vector control: ${ }^{\star \star} P<0.01,{ }^{\star \star *} P<0.001$.

\section{miR-24-1-5p regulates $\beta$-catenin}

The target genes that miR-24-1-5p may bind to were predicted using the bioinformatics method, miRanda version 3.3a, with default parameters and cutoffs (score $S \geq 140$ and energy $E \leq-7 \cdot 0$ ). $\beta$-Catenin was selected as a potential target among all of the candidates after considering the important role that $\beta$-catenin plays in CRC development. A binding domain was detected at the $3^{\prime}$-UTR of $\beta$-catenin where it might interact with miR-24-1-5p (Fig. 5(a)). Furthermore, to investigate the influence of miR-24-1-5p on $\beta$-catenin expression, miR-24-1-5p was overexpressed in human HCT-116 and Caco- 2 cells, and the changes in $\beta$-catenin level were measured by qRT-PCR and Western blot. In both cell lines, the protein level of $\beta$-catenin was significantly decreased whereas its mRNA level remained unchanged when miR-24-1-5p was overexpressed (Fig. 5(b) and (c)).

Further experiment was carried out in which HCT-116 and Caco-2 cells were transfected with miR-24-1-5p or the control vector for $24 \mathrm{~h}$, and cycloheximide $(10 \mu \mathrm{m})$ was then added to the cells for different time intervals before the cells were subjected to Western blot analysis to determine the protein synthesis rate of $\beta$-catenin. The result showed that the protein level of $\beta$-catenin in the cells transfected miR-24-1-5p plasmid was significantly decreased compared with the $\beta$-catenin level in the cells transfected with the control plasmid, indicating that miR-24-1-5p could promote the degradation of $\beta$-catenin protein to influence its stability. The result also suggested that miR-24-1-5p might negatively regulate $\beta$-catenin by directly binding to the $3^{\prime}$-UTR of the $\beta$-catenin mRNA.

\section{Genes associated with the wnt/ $\beta$-catenin pathway are regulated by $\mathrm{miR}-24-1-5 p$}

The impact of miR-24-1-5p over-expression on several downstream target genes of the $\beta$-catenin signalling pathway was also investigated. Changes in cyclin D1, c-Myc and CDK4 expression 
(a)

Forward : Score: $156 \cdot 000000$ Q:2 to $21 \mathrm{R:}: 1800$ to 1821 Align Len (19) $(73.68 \%)(84.21 \%)$

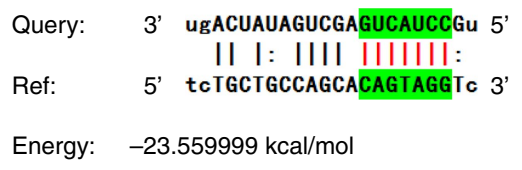

(b)
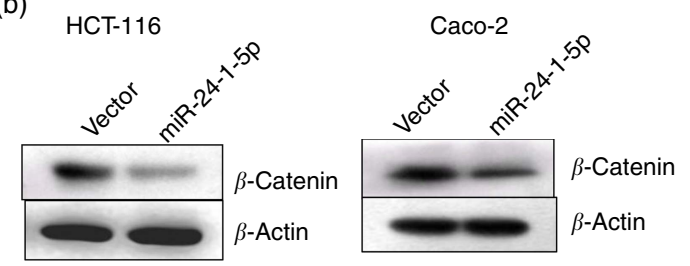

(c)

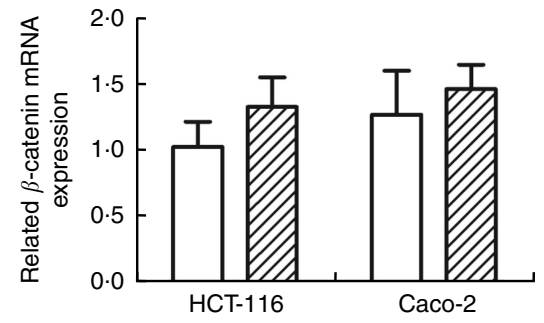

(d)

HCT-116

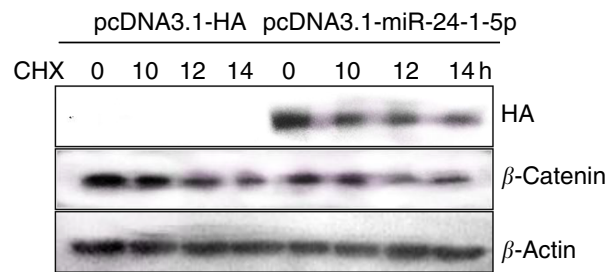

Caco-2

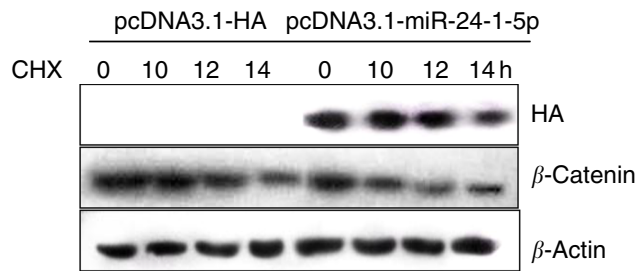

Fig. 5. miR-24-1-5p suppresses $\beta$-catenin expression via direct binding to the $3^{\prime}$-UTR (untranslated regions). (a) Sequence alignment of putative miR-24-1-5p binding sites in $\beta$-catenin 3'-UTR. (b) Western blot analysis of $\beta$-catenin protein level in HCT-116 and Caco-2 cells after being transfected with miR-24-1-5p. (c) Quantitative RTPCR analysis of $\beta$-catenin mRNA level in HCT-116 and Caco-2 cells after being transfected with miR-24-1-5p. (d) $\beta$-Catenin protein level after treatment with $10 \mu \mathrm{m}$ cycloheximide $(\mathrm{CHX})$ for different times $(0,10,12,14 \mathrm{~h})$. Differences within and between groups were compared by one-way ANOVA followed by Fisher's least significant difference analysis. $\square$, Vector; $\square$, miR-24-1-5p. HA, haemagglutinin.

levels in HCT-116 and Caco-2 cells were determined using Western blot and qRT-PCR. Both the mRNA and protein levels of these genes in HCT-116 and Caco- 2 cells decreased $24 \mathrm{~h}$ after they were transfected with miR-24-1-5p (Fig. 6(a) and (b)). Furthermore, other genes related to the $\mathrm{Wnt} / \beta$-catenin pathway such as SFRP2, SFRP5, E-cadherin, GSK3- $\beta, p-G S K 3-\beta$, and $B C L-2$ were also analysed. The expression of E-cadherin, p-GSK3- $\beta$ and SFRP5 was up-regulated, whereas the expression of BCL-2 was down-regulated, while no significant changes in expression were observed for GSK3- $\beta$ and SFRP2 (Fig. 6(c)). In summary, the data from in vitro human cancer cells demonstrated that $\beta$-catenin might be a direct target of miR-24-1-5p, mediating the effect of miR-24-1-5p by affecting the expression of its downstream target genes.

\section{Discussion}

Accumulated evidence suggests that the tightly regulated RNA network in a cell can be disrupted by aberrantly expressed miRNA, consequently triggering cancer development and metastasis. The importance of miRNA in various human cancers suggests that modulation of miRNA expression could be a novel strategy for cancer chemoprevention and therapy ${ }^{(36)}$. In the present study, miR-24-1-5p was significantly up-regulated in AOM/DSS-induced mice fed a diet containing BRB anthocyanins. Furthermore, mechanistic study indicated that miR-24-1-5p might play a tumour-suppressive role, and this was supported by its ability to inhibit cell proliferation, migration and survival, as well as colony formation when it was overexpressed in human CRC cells (Fig. 3). The tumour-suppressive role of miR-24-1-5p appeared to be mediated by its effect on the $\beta$-catenin signalling pathway (Figs. 4 and 5 ).

Implication of the Wnt $\beta$-catenin signalling pathway in cancer has been demonstrated by the effects of this signalling pathway on the growth, metabolism, angiogenesis, migration and metastasis of CRC cells ${ }^{(37)}$. Aberrant activation of $\beta$-catenin and its downstream target genes, which include $c-M y c$, cyclin D1 and $C D K 4$, can accelerate the initiation and progression of CRC. Our result clearly showed that in human CRC cells, these genes could be regulated through altering the expression of miR-24-1$5 p$. Indeed, over-expression of miR-24-1-5p in HCT-116 and Caco-2 cells down-regulated the expression of $\beta$-catenin, c-Myc, cyclin D1 and CDK4 (Fig. 6). Elucidating the cancer pathways and targets regulated by miR-24-1-5p via $\beta$-catenin would provide new insight into the process of oncogenesis for human CRC. Unfortunately, due to ethical issues we could not obtain direct evidence from human subjects to support our claim. However, further research is necessary to characterise the feasibility of targeting miR-24-1-5p in CRC as a cost-effective and simple therapeutic strategy for combating CRC at the clinical level. Much research has been devoted to BRB anthocyanins to investigate its usage as a chemoprevention agent for CRC. This aspect has been widely investigated in animal models and clinical patients. The mechanism underlying the 
(a)

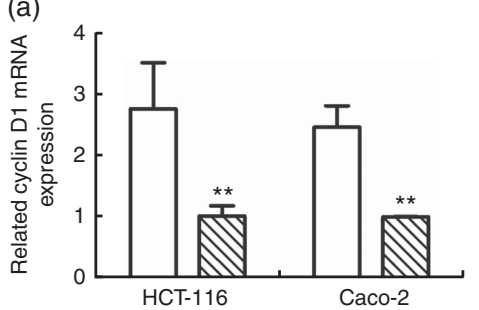

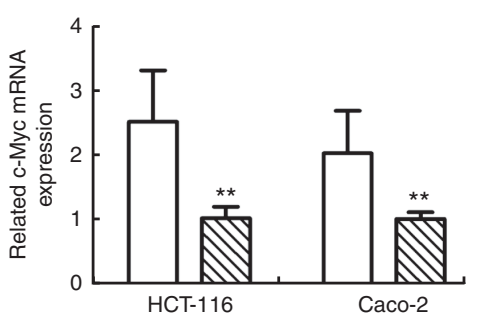

(c) HCT-116

(b) HCT-116

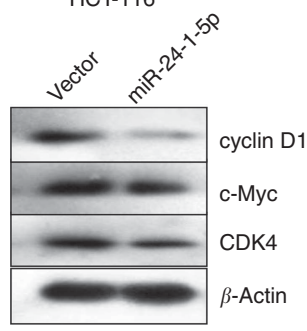

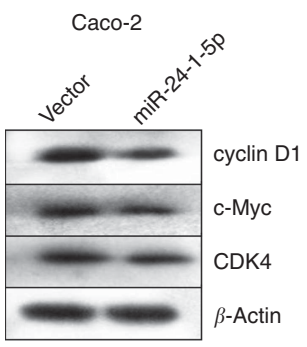

Fig. 6. Effects of miR-24-1-5p on genes associated with Wnt/ $\beta$-catenin signalling in colorectal cancer cells. Quantitative RT-PCR (a) or Western blot (b) analysis of cyclin D1, c-Myc and cyclin-dependent kinase 4 (CDK4) mRNA in HCT-116 and Caco-2 cells after transfection with $5 \mu \mathrm{g}$ miR-24-1-5p 24h (c) Western blot analysis of glycogen synthase kinase $3 \beta$ (GSK3- $\beta$ ), secreted frizzled related protein 2 (SFRP2), secreted frizzled related protein 5 (SFRP5), B-cell lymphoma-2 (BCL-2), E-cadherin and phospho-glycogen synthase kinase $3 \beta$ (p-GSK3- $\beta$ ) protein levels in HCT-116 and Caco-2 cells $24 \mathrm{~h}$ after transfection with miR-24-1-5p. Differences within and between groups were compared by one-way ANOVA followed by Fisher's least significant difference analysis. Mean values were significantly different when compared with the vector control: ${ }^{\star} P<0.05,{ }^{\star \star} P<0.01$. $\square$, Vector; $\mathbb{\Xi}$, miR-24-1-5p.

chemopreventive activity of BRB anthocyanins involves induction of cell apoptosis and differentiation, and reduced cell proliferation, inflammation, angiogenesis and invasiveness ${ }^{(38)}$. The chemopreventive effect of BRB anthocyanins on CRC also appears to involve the modification of gut microbiota and DNA demethylation $^{(39)}$. In this study, we have demonstrated for the first time that the chemopreventive effect of BRB anthocyanins against CRC might also involve the up-regulation of miR-24-1$5 \mathrm{p}$, and we have shown this through the use of a mouse model.

In summary, miR-24-1-5p might act as a tumour suppressor and modulate the growth of CRC cells through down-regulating the expression of $\beta$-catenin and its downstream target genes, and this could be part of the mechanism by which BRB anthocyanins manifest its chemopreventive effect against CRC. miR-24-1-5p could therefore be considered as a potential agent for the prevention or treatment of CRC in future.

\section{Acknowledgements}

This work was financially supported by National Science Foundation of China to X. B. (grant nos 81272333 and 81472821); programme of Liaoning Excellent Talents in University to X. B. (LETU no. LR 2014001) and in part from Innovation Team Project (no. LT2015011) from the Education Department of Liaoning Province. The authors thank Dr Alan K. Chang for revising the language of the manuscript.

H. Z. and J. G. designed, performed, analysed experiment and wrote the manuscript. L. M., Q. L., M. G., T. M. and Q. Z. performed and analysed experiments. X. B. conceived the study, designed experiments, reviewed and analysed data, and wrote and edited the manuscript.

The authors declare no competing financial interest.

\section{References}

1. Jemal A, Bray F, Center MM, et al. (2011) Global cancer statistics. CA Cancer J Clin 61, 69-90.

2. Jemal A, Siegel R, Xu J, et al. (2010) Cancer statistics, 2010. CA Cancer J Clin 60, 277-300.

3. Lin CC, Gray PJ, Jemal A, et al. (2015) Androgen deprivation with or without radiation therapy for clinically node-positive prostate cancer. J Natl Cancer Inst 107, djv119.

4. Schoen RE, Pinsky PF, Weissfeld JL, et al. (2012) Colorectalcancer incidence and mortality with screening flexible sigmoidoscopy. $N$ Engl J Med 366, 2345-2357.

5. Zauber AG, Winawer SJ, O'Brien MJ, et al. (2012) Colonoscopic polypectomy and long-term prevention of colorectalcancer deaths. $N$ Engl J Med 366, 687-696.

6. Pessaux P, Chenard MP, Bachellier P, et al. (2010) Consequences of chemotherapy on resection of colorectal liver metastases. J Visc Surg 147, e193-e201.

7. Srivastava K \& Srivastava A (2012) Comprehensive review of genetic association studies and meta-analyses on miRNA polymorphisms and cancer risk. PLOS ONE 7, e50966.

8. Tokarz P \& Blasiak J (2012) The role of microRNA in metastatic colorectal cancer and its significance in cancer prognosis and treatment. Acta Biochim Pol 59, 467-474.

9. Bhardwaj A, Singh S \& Singh AP (2010) MicroRNA-based cancer therapeutics: big hope from small RNAs. Mol Cell Pharmacol 2, 213-219.

10. Bartel DP (2004) MicroRNAs: genomics, biogenesis, mechanism, and function. Cell 116, 281-297. 
11. Cho WC (2010) MicroRNAs in cancer - from research to therapy. Biochim Biophys Acta 1805, 209-217.

12. Michael MZ, O'Connor SM, van Holst Pellekaan NG, et al. (2003) Reduced accumulation of specific microRNAs in colorectal neoplasia. Mol Cancer Res 1, 882-891.

13. Monzo M, Navarro A, Bandres E, et al. (2008) Overlapping expression of microRNAs in human embryonic colon and colorectal cancer. Cell Res 18, 823-833.

14. Tazawa H, Tsuchiya N, Izumiya M, et al. (2007) Tumorsuppressive miR-34a induces senescence-like growth arrest through modulation of the E2F pathway in human colon cancer cells. Proc Natl Acad Sci U S A 104, 15472-15477.

15. Bandres E, Bitarte N, Arias F, et al. (2009) microRNA-451 regulates macrophage migration inhibitory factor production and proliferation of gastrointestinal cancer cells. Clin Cancer Res 15, 2281-2290.

16. Guo H, Hu X, Ge S, et al. (2012) Regulation of RAP1B by miR-139 suppresses human colorectal carcinoma cell proliferation. Int J Biochem Cell Biol 44, 1465-1472.

17. Sun JY, Huang Y, Li JP, et al. (2012) MicroRNA-320a suppresses human colon cancer cell proliferation by directly targeting beta-catenin. Biochem Biophys Res Commun 420, 787-792.

18. Lagos-Quintana M, Rauhut R, Lendeckel W, et al. (2001) Identification of novel genes coding for small expressed RNAs. Science 294, 853-858.

19. Sun Q, Zhang Y, Yang G, et al. (2008) Transforming growth factor-beta-regulated miR-24 promotes skeletal muscle differentiation. Nucleic Acids Res 36, 2690-2699.

20. Guo Y, Fu W, Chen H, et al. (2012) miR-24 functions as a tumor suppressor in Hep2 laryngeal carcinoma cells partly through down-regulation of the S100A8 protein. Oncol Rep 27, 1097-1103.

21. Mishra PJ, Song B, Mishra PJ, et al. (2009) MiR-24 tumor suppressor activity is regulated independent of $\mathrm{p} 53$ and through a target site polymorphism. PLOS ONE 4, e8445.

22. Szczyrba J, Nolte E, Hart M, et al. (2013) Identification of ZNF217, hnRNP-K, VEGF-A and IPO7 as targets for microRNAs that are downregulated in prostate carcinoma. Int $\mathrm{J}$ Cancer 132, 775-784

23. Du WW, Fang L, Li M, et al. (2013) MicroRNA miR-24 enhances tumor invasion and metastasis by targeting PTPN9 and PTPRF to promote EGF signaling. J Cell Sci 126, $1440-1453$.

24. Lin SC, Liu CJ, Lin JA, et al. (2010) miR-24 up-regulation in oral carcinoma: positive association from clinical and in vitro analysis. Oral Oncol 46, 204-208.
25. Liu X, Wang A, Heidbreder CE, et al. (2010) MicroRNA-24 targeting RNA-binding protein DND1 in tongue squamous cell carcinoma. FEBS Lett 584, 4115-4120.

26. Qin W, Shi Y, Zhao B, et al. (2010) miR-24 regulates apoptosis by targeting the open reading frame (ORF) region of FAF1 in cancer cells. PLOS ONE 5, e9429.

27. Gu J, Ahn-Jarvis JH, Riedl KM, et al. (2014) Characterization of black raspberry functional food products for cancer prevention human clinical trials. J Agric Food Chem 62, 3997-4006.

28. Wang LS, Hecht SS, Carmella SG, et al. (2009) Anthocyanins in black raspberries prevent esophageal tumors in rats. Cancer Prev Res 2, 84-93.

29. Montrose DC, Horelik NA, Madigan JP, et al. (2011) Antiinflammatory effects of freeze-dried black raspberry powder in ulcerative colitis. Carcinogenesis 32, 343-350.

30. Pan P, Skaer CW, Stirdivant SM, et al. (2015) Beneficial regulation of metabolic profiles by black raspberries in human colorectal cancer patients. Cancer Prev Res 8, 743-750.

31. Coates EM, Popa G, Gill CI, et al. (2007) Colon-available raspberry polyphenols exhibit anti-cancer effects on in vitro models of colon cancer. $J$ Carcinog $\mathbf{6}, 4$.

32. Bi X, Fang W, Wang LS, et al. (2010) Black raspberries inhibit intestinal tumorigenesis in apc $1638^{+/-}$and Muc2 $2^{-/-}$mouse models of colorectal cancer. Cancer Prev Res 3, 1443-1450.

33. Xiao T, Guo Z, Bi X, et al. (2017) Polyphenolic profile as well as anti-oxidant and anti-diabetes effects of extracts from freeze-dried black raspberries. J Funct Foods 31, 179-187.

34. Stoner GD, Wang LS, Zikri N, et al. (2007) Cancer prevention with freeze-dried berries and berry components. Semin Cancer Biol 17, 403-410.

35. Bi X, Tong C, Dockendorff A, et al. (2008) Genetic deficiency of decorin causes intestinal tumor formation through disruption of intestinal cell maturation. Carcinogenesis 29, $1435-1440$.

36. Xuan Y, Yang H, Zhao L, et al. (2015) MicroRNAs in colorectal cancer: small molecules with big functions. Cancer Lett 360, 89-105.

37. Zhang H, Zhu YQ, Wu YQ, et al. (2014) Detection of promoter hypermethylation of Wnt antagonist genes in fecal samples for diagnosis of early colorectal cancer. World J Gastroenterol 20, 6329-6335.

38. Li J, Zhang D, Stoner GD, et al. (2008) Differential effects of black raspberry and strawberry extracts on BaPDE-induced activation of transcription factors and their target genes. Mol Carcinog 47, 286-294.

39. Chen L, Jiang B, Zhong C, et al. (2018) Chemoprevention of colorectal cancer by black raspberry anthocyanins involved the modulation of gut microbiota and SFRP2 demethylation. Carcinogenesis 39, 471-481. 